\title{
Maximum Length Sequence Brainstem Auditory Evoked Responses in Term Neonates Who Have Perinatal Hypoxia-Ischemia
}

\author{
ZE D. JIANG, DOROTHEA M. BROSI, XIAO M. SHAO, AND ANDREW R. WILKINSON \\ Neonatal Unit, Department of Paediatrics, John Radcliffe Hospital, University of Oxford, Headington, \\ Oxford OX3 9DU, United Kingdom [Z.D.J., D.M.B., A.R.W.]; and Neonatal Unit, Department of \\ Paediatrics, Children's Hospital, Shanghai Medical University, Shanghai 200032, China [X.W.S.]
}

\begin{abstract}
ABST
Maximum length sequence brainstem auditory evoked re-
sponse (BAER) was studied within the first week after birth in 28
term neonates who had perinatal hypoxia-ischemia, or asphyxia.
In the BAER recorded using conventional averaging techniques
(click rate $21 / \mathrm{s}$ ), the only abnormality was a slight increase in
III-V interval, in addition to an increase in wave latencies when
including those who had an elevated threshold $(t$ test, all $p<$
0.05). In the maximum length sequence BAER, however, both
the III-V and I-V intervals in the asphyxiated infants were
significantly increased at all the $91 / \mathrm{s}, 227 / \mathrm{s}, 455 / \mathrm{s}$, and particu-
larly $910 / \mathrm{s}$ click rates $(p<0.05-0.001$ ). The I-III interval was
also increased significantly at $455 / \mathrm{s}$ and $910 / \mathrm{s}$ click rates (both
$p<0.05$ ). Wave V amplitude was significantly reduced at all the
click rates used (ANOVA, $p<0.05-0.001)$, particularly at
$910 / \mathrm{s}$, which sometimes was the only abnormality indicative of
brain damage. Both the amplitude ratios V/I and V/III were
significantly decreased at $455 / \mathrm{s}$ and $910 / \mathrm{s}$ click rates $(p<0.01$ or
\end{abstract}
ABSTRACT

0.001). A general trend was that BAER abnormalities after hypoxia-ischemia became more prominent as click rate was increased. Significant abnormalities occurred mainly at very high click rates (455/s and 910/s), which can be achieved using the maximum length sequence technique but not by using conventional averaging techniques. Thus, this technique, which can be used at the cribside, appears to be a better method for the early detection of brain damage after hypoxia-ischemia than using conventional averaging techniques, enhancing the diagnostic value of the BAER. (Pediatr Res 48: 639-645, 2000)
Abbreviations
BAER, brainstem auditory evoked response
nHL, normal hearing level
HI, hypoxia-ischemia
MLS, maximum length sequence

HI, or asphyxia, remains an important cause of perinatally acquired brain damage in term babies with subsequent lifelong sequelae (1). About half of term babies who have major symptoms of HI after asphyxia will die or sustain severe disability (2). Early intervention with neuroprotective and therapeutic measures is important for improving their outcome. This largely relies on early detection of neuropathology after HI. One of the currently available tests is BAER (3-7). Information derived from the BAER can predict cranial ultrasonographic abnormalities in neonates at risk for brain injury (6). However, the BAER has only modest association with neurologic status, and false-negative results are common (7). Babies with neurologic deficits often do not show any apparent abnormalities in the BAER. Thus, for babies with a normal

Received November 30, 1999; accepted May 3, 2000.

Correspondence: Dr. Ze Dong Jiang, Neonatal Unit, Department of Paediatrics, John Radcliffe Hospital, Headington, Oxford OX3 9DU, United Kingdom.

Supported by the Wellcome Trust, R\&D Anglia and Oxford Regional Health Authority, Children Nationwide Medical Research Fund, and an Allen \& Hanburys Research Award to Z.D. Jiang by the Royal College of Paediatrics and Child Health, U.K.
BAER, we cannot rule out the possibility of early or subtle brain damage.

The method of increasing stimulus rate while recording the BAER has been suggested by some investigators to be a stimulus challenge test or stress test for detecting certain neuropathology that cannot be demonstrated by the BAER elicited with conventional stimulus rates (usually $11 / \mathrm{s}$ or $21 / \mathrm{s}$; $(3,8-14)$. Our previous studies of the BAER in babies with various clinical conditions that may affect the CNS, such as asphyxia and meningitis $(9,15,16)$, suggest a possibility that stimulus rates higher than $91 / \mathrm{s}$ may be more effective in detecting neuropathology in the brain. However, the increase in stimulus rate is limited by the need to prevent responses from overlapping one other. Conventional evoked potential instruments, or averagers, use stimuli separated by fixed interstimulus intervals (time intervals between the stimuli). The lower limit of these fixed intervals is determined by the duration of the electrophysiologic response. Conventional stimulation and recording techniques require that the brain wave response to one stimulus is completed before the delivery of the next 
stimulus. Stimulation before response completion results in overlapped waveforms, which are difficult to interpret. In the case of the BAER, response components last for $10-12 \mathrm{~ms}$ after stimulus onset, imposing a limit of $10-12 \mathrm{~ms}$ on the interpulse interval, corresponding to a rate of $80 / \mathrm{s}$ to $100 / \mathrm{s}$. Refractory periods of auditory neurons are $<10 \mathrm{~ms}$. Thus, a maximum stimulus rate of $100 / \mathrm{s}$ in conventional BAER limits the study of adaptive or recovery processes and the detection of functional abnormality in the CNS.

The use of pseudorandom pulse trains that are binary sequences, called MLS, as acoustic stimuli is a method to circumvent the rate limitation imposed by conventional averaging techniques. This relatively new technique uses patterned stimulus presentation rather than the uniformly spaced stimuli used in conventional BAER. The nature of the stimulus and newly developed processing technique make it unnecessary to wait for the response of each pulse to be completed before applying a new pulse, thus allowing the pulses to be delivered at rates of up to $1,000 / \mathrm{s}$ or even higher (15-22). Because the patterned sequences of stimuli are generated by the averaging computer, this information is then used to perform online deconvolution (separation, alignment, and averaging) of overlapping individual responses. As in recording of conventional BAER, each waveform of the response is filtered and the waveforms are averaged. The final MLS BAER is then obtained by mathematically cross-correlating the collected data with a recovery sequence. Therefore, this technique permits the overlap of responses to successive stimuli and allows presentation of stimuli at much higher rates than is possible using conventional methods. Because the higher rates provide a much stronger physiologic challenge to auditory neurons and permit a more exhaustive sampling of physiologic recovery or fatigue than is possible using conventionally used rates, this technique can potentially improve the sensitivity of the BAER to detect neuropathology.

We have recently studied the MLS BAER in babies and found that this technique has the potential to improve the early detection of perinatal brain damage $(15,16,22)$. The present study aimed to examine whether the MLS BAER can improve the early diagnostic value of the BAER for brain damage in term neonates who had perinatal HI.

\section{METHODS}

\section{Subjects}

Study group. Twenty-eight newborn infants who had perinatal HI, 16 boys and 12 girls, were recruited from the intensive care unit, Children's Hospital of Shanghai Medical University. Their gestational age ranged between 37 and $42 \mathrm{wk}$ $(39.5 \pm 1.7 \mathrm{wk})$. The diagnosis of perinatal $\mathrm{HI}$ or asphyxia was based on 1) clinical signs of HI (hypotonia with reduced or no spontaneous movements, increased threshold for primitive reflexes, lethargic or comatose appearance, absent or very weak suck response and requirement of tube feedings, and particularly seizure) and other signs of hypoxia, including frequent depression and failure to breathe spontaneously at birth $(1,2)$; and 2) depression of the Apgar score $(<7$ at $5 \mathrm{~min}$ ), which remains a widely used definition of perinatal asphyxia despite some arguments. These babies often had meconium staining of the amniotic fluid and an arterial blood $\mathrm{pH}<7.20$. Twenty-two had clear evidence of HI on cranial computed tomography or ultrasound scan. The exclusion criteria were congenital malformation and congenital or perinatal CNS infection.

Normal control group. Twenty-four healthy newborn infants, 11 boys and 13 girls, who had a gestational age between 37 and 41 wk $(39.1 \pm 1.4 \mathrm{wk})$ were recruited. Their birth weights were all above the 10th centile (23). All had an Apgar score $\geq 8$ at both 1 and $5 \mathrm{~min}$. None had any major perinatal conditions such as asphyxia, intrauterine or postnatal infection, or congenital abnormality. The monaural BAER thresholds, determined by conventional BAER (i.e. the BAER recorded using conventional averaging technique) at a click rate of $21 / \mathrm{s}$, were all $<20 \mathrm{~dB} \mathrm{nHL}$.

\section{Equipment and stimuli for recording the MLS BAER}

A Bravo Portable Evoked Potential System (Nicolet Biomedical Inc., Madison, U.S.A.) was used to record and analyze the MLS BAER. The stimuli used to elicit the MLS BAER were clicks of rarefaction polarity, which were generated by $100-\mu$ s rectangular pulses and delivered monaurally through a TDH 39 earphone. Repetition rate of the clicks is defined by the shortest time interval between adjacent stimuli. The clicks were presented at four rates, 91/s, 227/s, 455/s, and 910/s, equivalent to a minimum interpulse interval (the duration of the sequence) of $11.1,4.4,2.2$, and $1.1 \mathrm{~ms}$, respectively.

For the purpose of neurologic assessment, the intensity of stimulation used to elicit conventional BAER is usually between 60 and $80 \mathrm{~dB} \mathrm{nHL}$, the most frequently used one being $70 \mathrm{~dB}$ nHL. The repetition rates used to elicit the MLS BAER are much faster than those used in conventional BAER. Because of temporal integration, the perception of loudness of the stimulus trains increases as repetition rate is increased. An intensity of $80 \mathrm{~dB} \mathrm{nHL}$ is intolerable for subjects with normal hearing. Even $70 \mathrm{~dB}$ nHL tends to be too loud for some subjects, particularly when the test takes a long time. We therefore choose $60 \mathrm{~dB} \mathrm{nHL}$ as a routinely used intensity of stimulation. All subjects were tested at this intensity, although some were also tested at 70 and $80 \mathrm{~dB}$ nHL when the threshold was $\geq 20 \mathrm{~dB} \mathrm{nHL}$.

Conventional BAER was also recorded at 21/s to compare the results of BAER variables with those of the MLS BAER and at various intensities of clicks to determine the threshold, defined as the lowest intensity of clicks that produced visible, replicable wave $\mathrm{V}$.

\section{Procedures of recording the MLS BAER}

This study was approved by the Central Oxford Research Ethics Committee. Recording of the MLS BAER in the neonates was performed between 1 and $7 \mathrm{~d}$ after birth after obtaining informed consent of parents and the pediatrician in charge. The babies lay supine in their crib in either the intensive care unit (for study group) or in a quiet room (for control group). We previously recorded the MLS BAER in 14 babies in the two environments. No significant differences were found in the recordings between the two environments. Recording of 
the BAER was started after the babies fell asleep naturally, often after a feeding. They remained asleep throughout the recording session. The left ear was tested in all subjects, although the right ear was also tested in some cases.

Three gold-plated disk electrodes were placed at the middle forehead (positive), the ipsilateral earlobe (negative) and the contralateral earlobe (ground). Interelectrode impedances were maintained at $<10 \mathrm{k} \Omega$. The electrodes were connected to an amplifier by shielded wires. Brain electrical activity in response to the click stimulation was amplified and filtered with a bandpass between 100 and $3,000 \mathrm{~Hz}$ before inputting to the averager. During the sweep, the system monitored the amplitude of incoming data and compared it against the sensitivity setting. If the data exceeded $91 \%$ of the sensitivity variable setting $(51 \mu \mathrm{V})$, that sweep (artifact) was automatically rejected. Both the ongoing filtered EEG and the running averaged BAER were monitored while averaging. Sampling was manually discontinued whenever there were excessive muscle artifacts on the monitoring oscilloscope. Brain responses to 1,500 clicks were averaged for each run. Two independent recordings were made in response to each stimulus condition. The repetition rate of the clicks was presented in the sequence of $91 / \mathrm{s}, 227 / \mathrm{s}, 455 / \mathrm{s}$, and $910 / \mathrm{s}$ in the first run. A reverse sequence was used in the second run. A sweep duration of 24 ms was used in recording the MLS BAER and a duration of 12 $\mathrm{ms}$ in conventional BAER.

\section{Data analyses}

Various BAER wave latencies, interpeak intervals, and amplitudes were measured and analyzed. We have previously noted that in very young infants, the down slope of wave I is affected greatly by the presence or absence of wave II, which varies considerably, or the place of wave II, if it is present. This often produces considerable variability of the amplitude of wave I, which in turn affects the reliability of diagnostic value of amplitude ratio V/I. Therefore, the amplitude of wave I was measured from its peak to the lowest trough between waves I and III. Because using the trough after wave III to measure the amplitude of wave III is less reliable, this amplitude was measured from the lowest trough between waves I and III to the peak of wave III, as reported by others (19). Measurement of the amplitude of wave $\mathrm{V}$ was made from the positive peak to the negative trough immediately after.

Mean measurements of two replicated MLS BAER recordings to each stimulus condition were used for data analyses. To keep the consistency of recording and analyzing conditions, only the data from the left ear are presented. All quantitative analyses of BAER variables were based on the data collected at the click intensity of $60 \mathrm{~dB} \mathrm{nHL}$ in the normal controls, and at $60 \mathrm{~dB} \mathrm{nHL}$ (for thresholds $<20 \mathrm{~dB} \mathrm{nHL}$ ) or $70 \mathrm{~dB} \mathrm{nHL}$ (for thresholds at $20-30 \mathrm{~dB} \mathrm{nHL}$ ) in the study group so that all data were analyzed at level $\geq 40 \mathrm{~dB}$ above the thresholds, as in the normal control group.

Mean and SD of each BAER variable at each stimulus condition were calculated for the study group, which were compared with the normal controls using $t$ test for latency and interpeak interval variables and ANOVA for amplitude variables because of the relatively greater variability.

\section{RESULTS}

Of the 28 asphyxiated infants, three had a significant elevation in BAER threshold ( $>30 \mathrm{~dB} \mathrm{nHL})$. Means and SD for each BAER variable at different click rates are given in Tables 1 (wave latencies and interpeak intervals) and 2 (amplitude variables).

Conventional BAER. Compared with those in the normal controls, the group means of BAER wave I, III, and V latencies

Table 1. Latencies and interpeak intervals of MLS BAER (click rate 91-910/s) and conventional BAER (21/s) in term asphyxiated neonates within first week after birth

\begin{tabular}{|c|c|c|c|c|c|c|}
\hline $\begin{array}{c}\text { BAER } \\
\text { variables }\end{array}$ & Subjects & $21 / \mathrm{s}$ & $91 / \mathrm{s}$ & $227 / \mathrm{s}$ & $455 / \mathrm{s}$ & $910 / \mathrm{s}$ \\
\hline \multirow[t]{3}{*}{$\mathrm{I}(\mathrm{ms})$} & Normal & $2.32 \pm 0.16$ & $2.44 \pm 0.20$ & $2.62 \pm 0.19$ & $2.79 \pm 0.18$ & $2.85 \pm 0.25$ \\
\hline & Asphyxia† & $2.60 \pm 0.43^{*}$ & $2.57 \pm 0.31$ & $2.72 \pm 0.32$ & $2.78 \pm 0.31$ & $2.84 \pm 0.30$ \\
\hline & Asphyxiat & $2.34 \pm 0.19$ & $2.34 \pm 0.16$ & $2.52 \pm 0.19$ & $2.58 \pm 0.18^{* *}$ & $2.67 \pm 0.19 *$ \\
\hline \multirow[t]{3}{*}{ III (ms) } & Normal & $5.12 \pm 0.21$ & $5.33 \pm 0.22$ & $5.68 \pm 0.25$ & $6.00 \pm 0.24$ & $6.08 \pm 0.31$ \\
\hline & Asphyxia† & $5.37 \pm 0.48^{*}$ & $5.48 \pm 0.42$ & $5.87 \pm 0.47$ & $6.15 \pm 0.40$ & $6.26 \pm 0.54$ \\
\hline & Asphyxiał & $5.15 \pm 0.24$ & $5.28 \pm 0.25$ & $5.63 \pm 0.30$ & $5.95 \pm 0.30$ & $6.07 \pm 0.29$ \\
\hline \multirow[t]{3}{*}{$\mathrm{V}(\mathrm{ms})$} & Normal & $7.29 \pm 0.20$ & $7.56 \pm 0.20$ & $8.22 \pm 0.25$ & $8.83 \pm 0.27$ & $8.92 \pm 0.30$ \\
\hline & Asphyxia & $7.65 \pm 0.57 *$ & $7.88 \pm 0.51 *$ & $8.57 \pm 0.54 *$ & $9.15 \pm 0.47 *$ & $9.30 \pm 0.51 *$ \\
\hline & Asphyxia & $7.46 \pm 0.38$ & $7.70 \pm 0.40$ & $8.39 \pm 0.43$ & $9.02 \pm 0.44$ & $9.16 \pm 0.48$ \\
\hline \multirow[t]{3}{*}{ I-III (ms) } & Normal & $2.79 \pm 0.18$ & $2.89 \pm 0.22$ & $3.07 \pm 0.24$ & $3.20 \pm 0.21$ & $3.22 \pm 0.28$ \\
\hline & Asphyxia† & $2.77 \pm 0.19$ & $2.92 \pm 0.20$ & $3.15 \pm 0.22$ & $3.37 \pm 0.24 *$ & $3.39 \pm 0.23 *$ \\
\hline & Asphyxiat & $2.80 \pm 0.21$ & $2.94 \pm 0.20$ & $3.12 \pm 0.23$ & $3.38 \pm 0.26^{*}$ & $3.40 \pm 0.22 *$ \\
\hline \multirow[t]{3}{*}{$\mathrm{III}-\mathrm{V}(\mathrm{ms})$} & Normal & $2.21 \pm 0.10$ & $2.22 \pm 0.15$ & $2.54 \pm 0.10$ & $2.83 \pm 0.21$ & $2.84 \pm 0.14$ \\
\hline & Asphyxia $\dagger$ & $2.27 \pm 0.16$ & $2.40 \pm 0.15^{* *}$ & $2.70 \pm 0.16^{* *}$ & $3.00 \pm 0.20 * *$ & $3.06 \pm 0.20 * * *$ \\
\hline & Asphyxia & $2.31 \pm 0.18 *$ & $2.40 \pm 0.20 * *$ & $2.76 \pm 0.20 * *$ & $3.06 \pm 0.25 * *$ & $3.08 \pm 0.27 * * *$ \\
\hline \multirow[t]{3}{*}{$\mathrm{I}-\mathrm{V}(\mathrm{ms})$} & Normal & $4.99 \pm 0.20$ & $5.12 \pm 0.22$ & $5.61 \pm 0.28$ & $6.05 \pm 0.32$ & $6.07 \pm 0.31$ \\
\hline & Asphyxia $\dagger$ & $5.06 \pm 0.29$ & $5.31 \pm 0.30 *$ & $5.85 \pm 0.33 *$ & $6.37 \pm 0.34^{* *}$ & $6.45 \pm 0.34 * * *$ \\
\hline & Asphyxiat & $5.11 \pm 0.34$ & $5.36 \pm 0.34 *$ & $5.88 \pm 0.39 *$ & $6.44 \pm 0.40 * *$ & $6.49 \pm 0.43 * * *$ \\
\hline
\end{tabular}

Values are mean $\pm \mathrm{SD}$.

$* p<0.05 ; * * p<0.01 ; * * * p<0.001 ; t$ test comparing asphyxia groups with normal group.

$\dagger$ Asphyxia $(n=28)$, including three infants who had a significantly elevated threshold ( $>30 \mathrm{~dB}$ nHL).

Asphyxia $(n=25)$, excluding the three infants. 
Table 2. Amplitude variables of MLS BAER (click rate 91-910/s) and conventional BAER (21/s) in term asphyxiated neonates within first week after birth

\begin{tabular}{|c|c|c|c|c|c|c|}
\hline $\begin{array}{c}\text { BAER } \\
\text { variables }\end{array}$ & Subjects & $21 / \mathrm{s}$ & $91 / \mathrm{s}$ & $227 / \mathrm{s}$ & $455 / \mathrm{s}$ & $910 / \mathrm{s}$ \\
\hline \multirow[t]{2}{*}{$\mathrm{I}(\mu \mathrm{V})$} & Normal & $0.196 \pm 0.055$ & $0.141 \pm 0.038$ & $0.093 \pm 0.024$ & $0.060 \pm 0.017$ & $0.034 \pm 0.013$ \\
\hline & Asphyxia† & $0.195 \pm 0.054$ & $0.130 \pm 0.046$ & $0.094 \pm 0.034$ & $0.059 \pm 0.017$ & $0.032 \pm 0.013$ \\
\hline \multirow[t]{3}{*}{ III $(\mu \mathrm{V})$} & Normal & $0.219 \pm 0.057$ & $0.181 \pm 0.052$ & $0.127 \pm 0.037$ & $0.086 \pm 0.024$ & $0.047 \pm 0.016$ \\
\hline & Asphyxia $\dagger$ & $0.206 \pm 0.059$ & $0.151 \pm 0.048$ & $0.107 \pm 0.040$ & $0.069 \pm 0.019^{*}$ & $0.044 \pm 0.015$ \\
\hline & Asphyxiał & $0.218 \pm 0.051$ & $0.152 \pm 0.040$ & $0.103 \pm 0.024^{*}$ & $0.069 \pm 0.016^{*}$ & $0.044 \pm 0.011$ \\
\hline $\mathrm{V}(\mu \mathrm{V})$ & Asphyxia & $0.195 \pm 0.068$ & $0.143 \pm 0.045^{*}$ & $0.091 \pm 0.030^{* *}$ & $0.055 \pm 0.019^{* *}$ & $0.027 \pm 0.009^{* * *}$ \\
\hline \multirow[t]{3}{*}{$\mathrm{V} / \mathrm{I}$} & Normal & $1.138 \pm 0.529$ & $1.380 \pm 0.628$ & $1.409 \pm 0.481$ & $1.452 \pm 0.429$ & $1.605 \pm 0.728$ \\
\hline & Asphyxia† & $0.967 \pm 0.416$ & $1.355 \pm 1.056$ & $1.279 \pm 0.881$ & $0.963 \pm 0.409 * *$ & $1.025 \pm 0.477 * *$ \\
\hline & Asphyxia & $1.028 \pm 0.470$ & $1.158 \pm 0.637$ & $1.204 \pm 0.959$ & $0.892 \pm 0.358 * * *$ & $1.043 \pm 0.547 * *$ \\
\hline \multirow[t]{2}{*}{ V/III } & Normal & $0.933 \pm 0.230$ & $1.021 \pm 0.254$ & $1.018 \pm 0.228$ & $1.002 \pm 0.218$ & $1.194 \pm 0.542$ \\
\hline & Asphyxia $†$ & $0.878 \pm 0.221$ & $0.995 \pm 0.399$ & $1.034 \pm 0.358$ & $0.788 \pm 0.281 * *$ & $0.735 \pm 0.336^{* *}$ \\
\hline
\end{tabular}

Values are mean $\pm \mathrm{SD}$.

$* p<0.05 ; * *<<0.01 ; * * * p<0.001$; ANOVA comparing asphyxiated groups with normal group.

$\uparrow$ Asphyxia $(n=28)$, including three infants who had a significantly elevated threshold $(>30 \mathrm{~dB} \mathrm{nHL})$.

$\$$ Asphyxia $(n=25)$, excluding the three infants.

at $21 / \mathrm{s}$ click rate were increased in the 28 asphyxiated babies (all $p<0.05$, Table 1). However, this increase is largely related to an elevated threshold in a few of these babies. After excluding the data of the three asphyxiated babies whose threshold were $>30 \mathrm{~dB} \mathrm{nHL}$, there were no significant differences between the two groups of babies in any of these latencies. No difference was found between the asphyxiated and normal infants in all interpeak intervals (I-III, III-V, and $\mathrm{I}-\mathrm{V})$, although III-V interval was slightly increased when excluding the three asphyxiated babies with a significantly elevated threshold ( $\geq 30 \mathrm{~dB} \mathrm{nHL} ; p<0.05$; Table 1). There were also no differences between the two groups of babies in all amplitude variables, including wave I, III, and V amplitudes and amplitude ratios V/I and V/III (Table 2). Very few babies showed abnormalities in the central components of conventional BAER, even though some of the babies were in a very poor clinical condition.

MLS BAER. Recording of the BAER using the MLS technique was successfully performed in all babies, with no particular difficulties. Satisfactory recordings were always achieved in a sleeping baby. Major electrical interference occurred very occasionally.

BAER waveform changes with varying click rate in the asphyxiated babies were generally similar to those in the normal control infants. As click rate was increased, all wave latencies were increased and wave amplitude reduced. Figure 1 shows MLS BAER recordings in a normal term neonate (Fig $1 A$ ) and a term neonate who had severe, prolonged perinatal HI (Fig 1B). At 91/s click rate, the waveform of the asphyxiated infant is very similar to that of the normal infant except for a slightly increased $\mathrm{I}-\mathrm{V}$ interpeak interval. As click rate is increased, interpeak intervals, mainly III-V interval, are significantly increased $(>2 \mathrm{SD}$ above the mean in normal controls), and wave amplitude, particularly wave V amplitude, is significantly reduced $(<5$ th centile in normal controls). This infant had a completely normal waveform in conventional BAER.

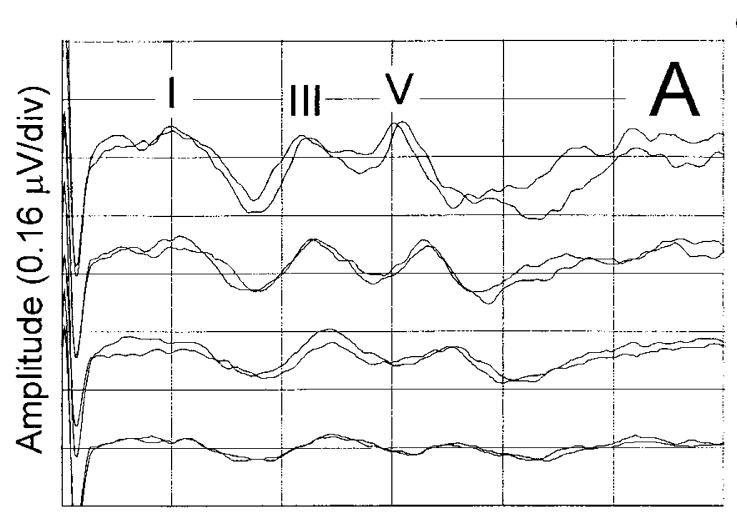

Click rate (/sec)

91

227

455

910

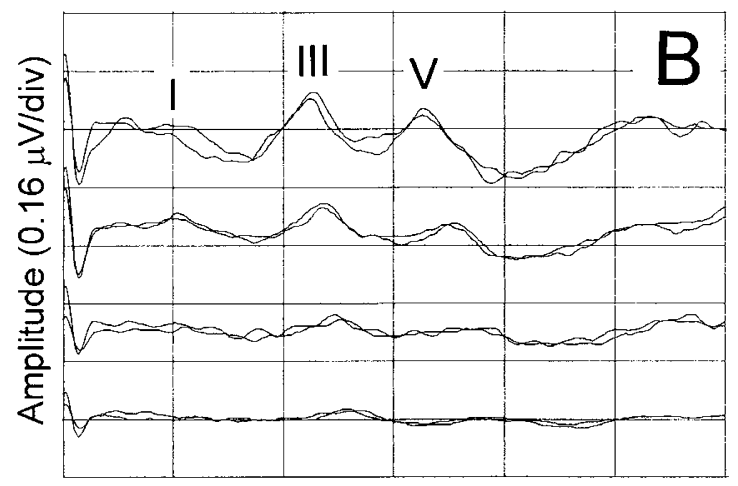

91

227

910

\section{Time (2.4 ms/div)}

Figure 1. Sample recordings of the MLS BAER in a normal term neonate $(A)$ and a term neonate who had perinatal HI $(B)$.

Waveform morphology. As we reported previously (22), waves I, III, and V were often well defined, although wave I sometimes became less distinct at 455/s and 910/s. Both waves II and IV were difficult to identify in most cases. The III-V complex, defined by the BAER components between the rapid increment in amplitude to the peak of wave III and the rapid decrement in amplitude after the peak of wave $\mathrm{V}$, was the most 
easily identifiable and reliable portion of the BAER waveform at all click rates used in this study.

Latencies. No differences in wave I latency were found between the 28 asphyxiated babies and the normal control infants. The increment of wave I latency with increasing click rate in the asphyxiated infants was relatively smaller. After excluding the data from the three asphyxiated babies with a threshold $>30 \mathrm{~dB} \mathrm{nHL}$, the group mean of wave I latency became shorter at 455/s and 910/s click rates $(p<0.01$ and 0.05 ), suggesting that the rate-dependent change in wave I latency after $\mathrm{HI}$ is decreased.

The means of wave $\mathrm{V}$ latency in the 28 asphyxiated infants were significantly increased at all four click rates used $(p<$ 0.05-0.001). After excluding the data from the three asphyxiated babies with a threshold $>30 \mathrm{~dB} \mathrm{nHL}$, this latency was still slightly increased, although statistical significance was found only at the click rate of 910/s (Table 1). Wave III latency did not differ significantly between the two groups of subjects at all click rates used.

Interpeak intervals. In general, the abnormalities in the intervals in the asphyxiated infants were increased with the increase in click rate. The I-III interval was slightly increased at the click rates used. Statistical significance was only found at the very high click rates (455/s and 910/s, whether including or excluding the three babies who had an elevated threshold; Table 1). The slope of the I-III interval-rate function was significantly steeper in the asphyxiated babies than in the normal control infants (one-sample $t$ test, $p<0.01$ ). The III-V interpeak interval was significantly increased at all four click rates used $(p<0.01-0.001)$. The $\mathrm{I}-\mathrm{V}$ interval was also increased at all click rates $(p<0.05-0.001)$. The increase in both III-V and I-V intervals was most significant at 910/s $(p<$ $0.001)$.

No differences in the I-III, III-V, and I-V intervals were found between the means of the 28 asphyxiated babies and those of the 25 asphyxiated babies (i.e. excluding those with an elevated threshold; Table 1). This can be explained by the fact that BAER intervals are generally not affected by the change in threshold or stimulus intensity (12).

Amplitudes. There were no differences in wave I amplitude between the asphyxiated and normal infants at all four click rates (Table 2). Wave III amplitude was slightly reduced in the asphyxiated babies, whether including or excluding the data from the three babies who had a significantly elevated threshold.

Of the BAER wave amplitudes, only wave $\mathrm{V}$ amplitude was abnormally, significantly reduced in the asphyxiated babies at all click rates used ( $p<0.05-0.001$; Table 2$)$, although this reduction was increased with the increase in click rate. Compared with the measurements in the normal infants, wave $\mathrm{V}$ amplitude in the asphyxiated babies was reduced by $23.4 \%$ at $91 / \mathrm{s}, 26.0 \%$ at $227 / \mathrm{s}, 32.9 \%$ at $455 / \mathrm{s}$, and $47.1 \%$ at $910 / \mathrm{s} \mathrm{click}$ rates (excluding the three babies with an elevated threshold; similar case was also seen when including these babies). In five asphyxiated babies, an abnormal, significant reduction of wave $\mathrm{V}$ amplitude $(<5$ th centile in normal infants) at the highest click rate $(910 / \mathrm{s})$ was the only abnormality that could be detected in the MLS BAER.
The amplitude ratio V/I, a frequently used amplitude variable, in the asphyxiated babies was decreased slightly at $91 / \mathrm{s}$ and $227 / \mathrm{s}$ click rates but significantly at $455 / \mathrm{s}$ and $910 / \mathrm{s}$ click rates $(p<0.01-0.001)$, whether including or excluding those babies with an elevated threshold (Table 2). The amplitude ratio V/III has been rarely described in the literature. Group mean of this ratio in the normal controls was always approximately 1.0 across the various click rates, suggesting that in normal babies this ratio is not significantly affected by the change in click rate (Table 2). In the asphyxiated babies, the V/III ratio was not affected by increasing click rate up to $227 /$ s, but was decreased significantly as the rate was increased further, particularly at 910/s (Table 2).

Taken together, the differences in the BAER between the asphyxiated and normal control babies were much greater in the MLS BAER than in conventional BAER. There were more asphyxiated babies who had abnormalities in the MLS BAER (mainly at 455/s and 910/s) than those who had abnormalities in conventional BAER. The babies who had significant MLS BAER abnormalities were often associated with significant clinical signs of HI (Sarnat and Sarnat grade II or III) $(1,2)$, a low $\mathrm{PaO}_{2}$, a raised $\mathrm{PaCO}_{2}$, and metabolic acidosis (arterial blood $\mathrm{pH}<7.10$ ).

After the first week. Some of the asphyxiated infants were retested until discharge. The changes in the MLS BAER were generally associated with the severity of the clinical condition. The abnormalities in the MLS BAER tended to normalize with recovery some weeks later in most cases but persisted for longer in a few. In rare cases, the MLS BAER became worse in the second or third week.

Figure 2 shows MLS BAER recordings in a boy who had severe, prolonged HI, with significant clinical signs of $\mathrm{HI}$, frequent seizures, and an Apgar score of 3 at $5 \mathrm{~min}$. He was resuscitated after birth. The first recordings were made on the second day after birth (39 wk gestation). No abnormalities can be seen in the waveform at 91/s and 227/s click rates (as well as in conventional BAER waveform at $21 / \mathrm{s}$ ). The I-V interval and, in particular, III-V interval are significantly increased ( $>$ $2 \mathrm{SD}$ above the mean in normal infants), and the wave $\mathrm{V}$ amplitude is significantly reduced at $455 / \mathrm{s}$ and $910 / \mathrm{s}$ click rates $(<5$ th centile in normal infants). The second recordings were made on the 10th day after birth (40 wk conceptional age). The abnormalities are significantly improved (the intervals are shortened and wave amplitudes are increased at $455 / \mathrm{s}$ and 910/s click rates).
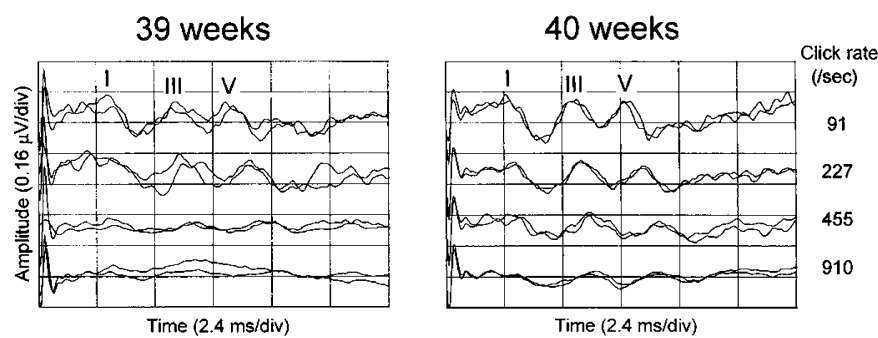

Figure 2. MLS BAER recordings, made on the second day after birth (39 wk gestation) and the 10th day (40 wk conceptional age), in a boy who had severe perinatal $\mathrm{HI}$. 


\section{DISCUSSION}

Implications of MLS BAER changes in the neonates with HI. Changes in the BAER with increasing stimulus presentation rate, or rate-dependent changes, primarily reflect neural processes concerning the efficacy of central synaptic transmission, as well as neural synchronization and metabolic status of auditory neurons in the brainstem after the presentation of a physiologic challenge $(19,24)$. Thus, based on the ratedependent BAER changes, the stimulus challenge test provides a possible means to study central synaptic efficacy after perinatal HI. Our study of the rate-dependent changes in normal term neonates suggests that synaptic efficacy in the neonatal brainstem auditory pathway is less mature than general function of the pathway $(10,22)$. A clinical implication of this relative immaturity is that synaptic efficacy of the brainstem auditory pathway may be more susceptible to unfavorable perinatal conditions than general function of the pathway is. By presenting a physiologic challenge to auditory neurons, the stimulus challenge test may be particularly useful to study central synaptic function and to detect some neuropathologic processes that may not be demonstrated by the BAER obtained using conventional low-rate stimulation.

Each of the BAER components requires the integrity of an anatomically diffuse system comprising a set of neurons, their axons, and the neurons on which they terminate. Disruption of any portion of the system will alter the amplitude or the latency of that component $(25,26)$. Abnormalities in the BAER after perinatal $\mathrm{HI}$ result from $\mathrm{HI}$ neuronal damage that may interfere with nerve conduction and synaptic transmission of the brain. The increase in the differences between the asphyxiated and normal term neonates in the MLS BAER with the increasing click rate reflects a decreased efficacy of central synaptic transmission or a decreased ability of central neurons to recover in time to transmit the next stimulus-evoked response after HI. In other words, there is an acute impairment, or reduction, of central synaptic efficacy. This impairment may result from the damage to neuronal function in the brain after HI. Asphyxia disturbs the metabolism of neurons and depresses the electrophysiologic function of synapses, which, in the developing brain, transmit developmental as well as regulatory signals between neurons. The efficacy of synaptic transmission, an important index of synaptic function, is related to the mechanisms for synthesis, release, and uptake of neurotransmitters that are known to play a crucial role in the regulation of neural development as distinct from their differentiated function as neural signal modulators. Significant HI causes excessive release of glutamate from the presynaptic transmitter site and compromises the uptake pumps of glutamate within the synaptic cleft so that the concentration of glutamate increases 20 -fold within minutes, leading to irreversible neuronal injury, which is particularly prominent in the developing human brain $(27,28)$. Inasmuch as glutamate, an excitatory amino acid neurotransmitter, mediates fast excitatory synaptic transmission, it is therefore possible that the efficacy of synaptic transmission in a baby's brain after HI is impaired. This impairment could be an early indicator of perinatal HI.
Amplitude reduction in evoked potentials may be caused by synaptic inefficiency or asynchronous firing of neurons after brain damage. The present study shows that the reduction of wave $\mathrm{V}$ amplitude with increasing click rate in the asphyxiated babies was much more significant than reduction of the amplitudes of waves I and III, particularly at $910 /$ s click rate. This is further confirmed by the significant decrease in the amplitude ratios V/I and V/III at the very high click rates in these babies. These findings suggest that the more central components of the BAER (wave $V$, generating from the rostral part of the brainstem auditory pathway) are affected by perinatal HI relatively more than the more peripheral components (waves I and III, generating from the more peripheral part of the pathway). It appears that, after HI, synaptic efficacy in the more central, or rostral, part of the brainstem is impaired more than the more peripheral part of the brainstem, or that the more central part of the brainstem is more vulnerable to a physiologic challenge.

MLS BAER-a promising technique to improve early detection of HI brain damage at cribside. In the past $2 \mathrm{y}$, we have studied the use of MLS BAER in neonates with various perinatal problems and have found that this response can be reliably recorded at the cribside in the neonatal intensive care unit, as well as in a quiet room, with good reproducibility (15, 16, 22). Abnormalities in the central components of the MLS BAER, including increased interpeak intervals and, in particular, reduced amplitude of wave V, occurred mainly at very high click rates (455/s and particularly 910/s). The abnormalities were predominantly seen in babies who had severe perinatal problems that may either directly or indirectly damage the neonatal brain, including $\mathrm{HI}$ and severe hyaline membrane disease. MLS BAER findings were correlated well with clinical conditions. By contrast, the central components of conventional BAER at 21/s were rarely abnormal.

In this study, no major abnormalities were found in the central components of the BAER, elicited using conventional averaging techniques at the routinely used $21 / \mathrm{s}$ click rate, in the asphyxiated babies. Therefore, the use of conventional BAER to detect early or subtle brain damage after $\mathrm{HI}$ is rather limited. With MLS BAER, the abnormalities increased with the increase in click rate. Significant BAER abnormalities were often seen only at very high rates of clicks (455/s and particularly 910/s), which can be achieved using the MLS technique but not using conventional averaging techniques. In particular, an abnormal, significant reduction of wave $\mathrm{V}$ amplitude, which was often associated with a significant decrease in the amplitude ratios V/I and V/III, at the highest click rate (910/s) sometimes was the only BAER abnormality indicative of neurologic impairment of the brain. It appears that this reduction is a useful index for early or subtle brain damage after HI. Those who had MLS BAER abnormalities were often associated with significant clinical signs of HI. By contrast, most central components of conventional BAER (21/s) in the asphyxiated babies fell within normal ranges, although some of these babies were in a very poor clinical condition. These findings indicate that, compared with conventional BAER, the MLS BAER is more valid in detecting neuropathology in the brainstem auditory pathway after perinatal HI. Thus, the MLS technique 
appears to be a better method for early detection of brain damage after $\mathrm{HI}$ than the conventional averaging technique, and appears to be a promising technique to enhance the diagnostic value of the BAER.

Acknowledgments. The authors thank Dr. Yun Cao in Shanghai Medical University for assisting in the collection of part of the data. We also thank the nurses and pediatricians at the Neonatal Unit of the Department of Paediatrics and the Department of Obstetrics and Gynaecology for their assistance in the recruitment of subjects.

\section{REFERENCES}

1. Evans DJ, Levene MI 1999 Hypoxic-ischaemic injury. In: Rennie JM, Roberton NRC (eds) Textbook of Neonatology. Churchill-Livingstone, Edinburgh, pp 1231-1251

2. Levene MI 1992 The asphyxiated newborn infant. In: Levene MI, Bennett MJ, Punt J (eds) Fetal and Neonatal Neurology and Neurosurgery. Churchill-Livingstone, Edinburgh, pp 405-425

3. Hecox K, Cone B, Blaw M 1981 Brainstem auditory evoked response in the diagnosis of pediatric neurologic diseases. Neurology (Ny) 31:832-839

4. Jiang ZD 1998 Maturation of peripheral and brainstem auditory function in the firs year following perinatal asphyxia: a longitudinal study. J Speech Lang Hear Res 41:83-93

5. Jiang ZD, Tierney TS 1996 Long-term effect of perinatal and postnatal asphyxia on developing human auditory brainstem responses: brainstem impairment. Int J Pediatr Otorhinolaryngol 34:111-127

6. Karmel BZ, Gardner JM, Zappulla RA, Magnano CL, Brown EG 1988 Brain-stem auditory evoked responses as indicators of early brain insult. Electroencephalogr Clin Neurophysiol 71:429-442

7. Majnemer A, Rosenblatt B, Riley P 1988 Prognostic significance of the auditory brainstem evoked response in high-risk neonates. Dev Med Child Neurol 30:43-52

8. Jiang ZD, Tierney TS 1996 Binaural interaction in human neonatal auditory brainstem. Pediatr Res 39:708-714

9. Jiang ZD 1998 Outcome of brainstem auditory electrophysiology in children who survived purulent meningitis. Ann Otol Rhinol Laryngol 108:429-434

10. Jiang ZD, Brosi DM, Wilkinson AR 1998 Immaturity in electrophysiological response of the neonatal auditory brainstem to high repetition rates of click stimulation. Early Hum Dev 52:133-143

11. Henderson-Smart DJ, Pettigrew AG, Edwards DA, Jiang ZD 1991 Brain stem auditory evoked responses: physiological and clinical issues. In: Hanson MA (ed) The Fetal and Neonatal Brain Stem: Developmental and Clinical Issues. Cambridge University Press, Cambridge, pp 211-229
12. Chiappa KH 1990 Brainstem auditory evoked potentials: methodology. In: Chiappa $\mathrm{KH}$ (ed) Evoked Potentials in Clinical Medicine. Raven Press, New York, pp $173-221$

13. Gerling IJ, Finitzo-Hieber T 1983 Auditory brainstem response with high stimulus rates in normal and patient populations. Ann Otol Rhinol Laryngol 92:119-123

14. Pratt H, Ben-David Y, Peled R, Podoshin L, Scharf B 1981 Auditory brain stem evoked potentials: clinical promise of increasing stimulus rate. Electroencephalog Clin Neurophysiol 51:80-90

15. Jiang ZD, Brosi DM, Wilkinson AR 1998 Maximum length sequence brainstem evoked response: a potentially sensitive means to detect neural dysfunction of the brain in high risk neonates. Abstract 290 in Final Programme of 19th Annual Conference of the European Society for Pediatric Research, pp 97

16. Jiang ZD, Brosi DM, Wilkinson AR 1999 Detection of auditory impairment in high-risk infants using MLS BAER. Pediatr Res 45:343A(abstr)

17. Eysholdt U, Schreiner C 1982 Maximum length sequences: a fast method for measuring brainstem evoked responses. Audiology 21:242-250

18. Burkard R, Shi Y, Hecox KE 1990 Brainstem auditory evoked responses elicited by maximum length sequences: effects of simultaneous masking noise. J Acoust Soc Am $87: 1665-1672$

19. Lasky RE 1997 Rate and adaptation effects on the auditory evoked brainstem response in human newborns and adults. Hear Res 111:165-176

20. Lasky RE, Perlman J, Hecox KE 1992 Maximum length sequences auditory evoked brainstem responses in human newborns and adults. J Am Acad Audiol 3:383-389

21. Picton TW, Champagne SC, Kekkett AJ 1992 Human auditory evoked potentials recorded using maximum length sequences. Electroencephalog Clin Neurophysiol $84: 90-100$

22. Jiang ZD, Brosi DM, Wilkinson AR 1999 Neonatal brainstem auditory evoked response recorded using maximum length sequences. Biol Neonate 76:193-199

23. Yudkin PL, Aboualfa M, Eyre JA, Redman CWG, Wilkinson AR 1987 New birthweight and head circumference centile for gestational ages 24 to 42 weeks. Early Hum Dev 15:45-52

24. Ken-Dror A, Pratt H, Zeltzer M, Sujov P, Katzir J, Benderley A 1987 Auditory brainstem evoked potentials to clicks at different presentation rates: estimating maturation of pre-term and full-term neonates. Electroencephalog Clin Neurophysiol 68:209-218

25. Zaaroor M, Starr A 1991 Auditory brain-stem evoked potentials in cat after kainic acid induced neuronal loss: I. Superior olivary complex. Electroencephalog Clin Neurophysiol 80:422-435

26. Zaaroor M, Sarr A 1991 Auditory brain-stem evoked potentials in cat after kainic acid induced neuronal loss: II. Cochlear nucleus. Electroencephalog Clin Neurophysiol $80: 436-445$

27. Benveniste H, Drejer J, Schousboe A, Diemer NH 1984 Elevation of the extracellular concentrations of glutamate and aspartate in rat hippocampus during transient cerebral ischemia monitored by intracerebral microdialysis. J Neurochem 43:1369-1374

28. Represa A, Tremblay E, Ben-Ari Y 1989 Transient increase of NMDA-binding sites in human hippocampus during development. Neurosci Lett 99:61-66 